\title{
Nuevo código de puntuación de Gimnasia Artística Femenina Federación Internacional de Gimnasia Ciclo Olímpico 2022-2024
}

\author{
New code of points of Women Artistic Gymnastics \\ International Gymnastics Federation \\ Olympic Cycle 2022-2024
}

\section{* Claudia Marcela Perelman Barry}

Perelman, C. (2021). Nuevo código de puntuación de Gimnasia Artística Femenina. Federación Internacional de Gimnasia. Ciclo Olímpico 2022-2024. Revista Ciencias de la Actividad Física UCM, № 22(1), enero-junio, 1-10. DOI: http://doi.org/10.29035/rcaf.22.1.4

\section{RESUMEN}

El Código de Puntuación de la Federación Internacional de Gimnasia en las distintas disciplinas de la Gimnasia, sufre modificaciones y se actualiza después de cada Juego Olímpico; obligando a una constante actualización de los jueces y entrenadores. Estos cambios rigen para los 4 años que dura el ciclo olímpico, y se dan a conocer en los Cursos Internacionales de Jueces que imparte la Federación Internacional de Gimnasia para cada disciplina deportiva.

Por la pandemia que ha afectado al mundo, y por consecuencia al deporte, los Juegos Olímpicos de Tokio 2020 fueron pospuestos para el año 2021; y con esto, se ha postergado en un año la entrada en vigencia de los reglamentos de la Gimnasia del próximo ciclo olímpico, periodo que se extenderá extraordinariamente desde el año 2022 al 2024. El presente trabajo tiene como objetivo, dar a conocer los principales cambios que está proponiendo el Comité Técnico Femenino de la Federación Internacional de Gimnasia, en la primera edición del nuevo Código de Puntuación de la Gimnasia Artística Femenina para el ciclo olímpico 2022-2024.

Palabras clave: Gimnasia, gimnasia artística, gimnasia artística femenina, código de puntuación, Federación Internacional de Gimnasia.

\begin{abstract}
The International Gymnastics Federation's Code of Points, for different disciplines in Gymnastics, undergoes modifications and is updated after each Olympic Game, forcing a constant update of the judges and coaches. These changes apply for the 4 years of the Olympic cycle and are announced in the International Judges Courses, imparted by the International Gymnastics Federation for each sport discipline after the Olympics.

Because of how the pandemic has affected the world, and consequently sports, the Olympic Games of Tokyo 2020 were postponed to the year 2021. With this, the validity of Gymnastic regulations for the next Olympic cycle has been postponed by one year, so the cycle will extraordinarily extend from 2022 to 2024.

The objective of this article is to announce the main changes that the International Gymnastics Federation's Women's Technical Committee is proposing, in the first edition of the new Code of Points for the Women's Artistic Gymnastics cycle of 2022-2024.
\end{abstract}

Key words: Gymnastics, artistic gymnastics, women's artistic gymnastics, code of points, International Gymnastics Federation.

Maestría en Administración y Gestión Deportiva, Universidad Metropolitana de Ciencias de la Educación, Santiago, Chile. ID https://orcid.org/0000-0003-3997-6855 claudia.perelman@umce.cl 
Perelman, C. (2021). Nuevo código de puntuación de Gimnasia Artística Femenina. Federación Internacional de Gimnasia. Ciclo Olímpico $2022-2024$. Revista Ciencias de la Actividad Física UCM, N²2(1), enero-junio, 1-10. DOI: http://doi.org/10.29035/rcaf.22.1.4

\section{INTRODUCCIÓN}

El Código de Puntuación (COP) de la Federación Internacional de Gimnasia (FIG) en las distintas disciplinas de la Gimnasia, sufre modificaciones y se actualiza después de cada Juego Olímpico (JJOO). Estos cambios se publican en un nuevo COP y rigen por cuatro años, es decir, para todo el ciclo olímpico siguiente. (FIG, 2017), sin embargo, para estar al día de los cambios sobre procesos de capacitación permanente de la disciplina se generan programas interactivos o software que facilitan la comprensión técnica de las modificaciones que se producen entre cada ciclo olímpico (Perelman, 2020). Del mismo modo, la mesa de ayuda facilita importantemente la tarea de los jueces (Sacchi et al., 2019).

En relación a los cambios del COP, estos también pueden ocurrir después de cada competencia oficial FIG durante el ciclo olímpico en curso. Para este efecto, el Comité Técnico respectivo de la FIG publica todos los elementos nuevos presentados en estos eventos en un newsletter; y estos a su vez se agregan al COP que está vigente, es decir, no se espera hasta el cambio de ciclo olímpico para incluirlos (FIG, 2018; 2019).

Por otro lado, la vigencia de los jueces internacionales caduca con el término de cada ciclo olímpico, por lo que los jueces internacionales deben aprobar los Cursos Internacionales de Jueces que imparte la FIG después de cada JJOO, para así renovar su Brevet (carnet) de Juez Internacional por cuatro años más (Gimnasialatina, 2019).

Cabe señalar, que dada la pandemia que ha afectado al mundo y al deporte durante el año en curso, todos los eventos internacionales de la FIC del año 2020, así como los JJOO de Tokio 2020, han sido postergados para el año 2021. Como consecuencia, se pospusieron todos los cursos internacionales de jueces en un año; y con esta medida, también se postergó en un año la entrada en vigencia de los nuevos COPs en la Gimnasia. Es así como entonces, el próximo ciclo olímpico comenzará el año 2021 y no el año 2020

El presente trabajo tiene como objetivo dar a conocer los principales cambios que se están proponiendo por parte del Comité Técnico Femenino de la FIG para el nuevo COP de la Gimnasia Artística Femenina en su primera edición, que se publicó a mediados del año 2019; con el propósito de tener una idea de lo que se espera del ciclo olímpico 2022-2024 en este deporte.

\section{El código de puntuación de la Gimnasia Ar- tística Femenina}

Para una mejor comprensión de los cambios que se proponen para el próximo COP, se debe entender primero ¿cómo se ordenan los contenidos y capítulos en este reglamento? (Tabla 1):

Tabla 1

Orden de los contenidos y capítulos.

\section{PARTE I: REGLAS PARA LOS PARTICIPANTES EN COMPETENCIAS}

Sección 1: Propósito

Sección 2: Reglas para las Gimnastas

Sección 3: Reglas para los Entrenadores

Sección 4: Reglas para el Comité Técnico

Sección 5: Reglas y Estructura de los Jurados de Aparatos

PARTE II: EVALUACIÓN DE LOS EJERCICIOS

Sección 6: Determinación de la Nota

Sección 7: Reglas para la Nota $D$

Sección 8: Reglas para la Nota E

Sección 9: Directivas Técnicas

\section{PARTE III: APARATOS}

Sección 10: Salto

Sección 11: Paralelas Asimétricas

Sección 12: Viga de Equilibrio

Sección 13: Suelo

PARTE IV - V: Tabla de Elementos y Apéndices 
Perelman, C. (2021). Nuevo código de puntuación de Gimnasia Artística Femenina. Federación Internacional de Gimnasia. Ciclo Olímpico $2022-2024$. Revista Ciencias de la Actividad Física UCM, N²2(1), enero-junio, 1-10. DOI: http://doi.org/10.29035/rcaf.22.1.4

El Comité Técnico Femenino de la FIG ya publicó la primera edición del nuevo COP de Gimnasia Artística Femenina para el ciclo 2022-2024, pero no será sino hasta después de los JJOO de Tokio 2021 que se publicará el Código definitivo; por lo que estos cambios aún pueden sufrir modificaciones y deben ser confirmados en la edición definitiva a fines del año 2021.

A continuación, se presentan los cambios más importantes que fueron publicados en esta primera edición del nuevo COP de la Gimnasia Artística Femenina, respecto a los capítulos Parte I, II y III de este reglamento.

\section{Código de puntuación de Gimnasia Artística Femenina ciclo 2022-2024 \\ Parte I. Reglas para los participantes en competencias}

\section{Sección 2: Reglas para las Gimnastas Responsabilidades de la Gimnasta:}

A partir del próximo ciclo olímpico, se permitirá subir sólo $5 \mathrm{~cm}$ la altura de las barras de la Paralela Asimétrica para las gimnastas que toquen la colchoneta con los pies durante el ejercicio. Se debe informar a los organizadores en el momento de la inscripción nominativa y el Jurado Superior lo verificará durante el entrenamiento. En el Código actual se permite subir las barras $10 \mathrm{~cm}$ (FIG, 2017).

\section{Obligaciones de las Gimnastas:}

En el nuevo COP se agregó la regla que indica, que la gimnasta no puede abstenerse intencionalmente a ponerse de pie después de una caída del aparato, para evitar con esto que comience a correr el tiempo de caída de 10 segundos para Viga y de 30 segundos para Paralelas Asimétricas; tiempo que parte desde la que gimnasta se pone de pie luego de una caída del aparato. Se quiere evitar con esto que se haga mal uso del tiempo de caída estipulado: descansando, ajustando las cayeras, recibiendo magnesio del entrenador, retrasando la competencia sin justificación, etc.
En el punto que hace alusión a la obligación de la gimnasta de:

Evitar cualquier otro comportamiento indisciplinado o abusivo, o infringir aquellos de cualquier otro participante. Por ej. marcar el tapiz de suelo con magnesio, dañar cualquier superficie o parte de los aparatos durante la preparación de su ejercicio o quitar resortes del trampolín; la gimnasta corre/camina por debajo de $\mathrm{Bl}$ para comenzar su rutina (FIG, 2017, p.2).

Se incluye en el nuevo COP, que la gimnasta no puede usar agua para mojar la superficie de la Viga.

Respecto a la obligación de participar en la ceremonia de entrega de medallas, en el nuevo COP la gimnasta deberá presentarse con la vestimenta oficial de la delegación (buzo) de acuerdo al Protocolo de la FIG, y no con malla o leotardo como lo es actualmente.

En relación a la obligación de Indumentaria de Competencia, se incorpora que la gimnasta debe abstenerse de usar mangas/calcetines de compresión durante la competencia; así como la prohibición de pintarse la cara con cualquier maquillaje. De haber maquillaje, debe ser modesto y no retratar un personaje teatral (animal o humano).

\section{Sección 5: Reglas y Estructura de los Jurados de Aparatos Responsabilidades de los Jueces:}

En el nuevo COP se propone que sólo los jueces del Panel D (Dificultad) tengan la obligación de asistir al entrenamiento de podio. En el código actual es obligatorio para todos los jueces, es decir, tanto para el Panel D (Dificultad) como para el Panel E (Ejecución).

\section{Composición del Jurado del Aparato (Panel de Jueces):}

El nuevo COP plantea que los Panales de Jueces deberán estar conformados por 9 jueces, divididos en 2 jueces D (Dificultad) y 7 jueces E ejecución); para los distintos tipos de competencias, refiriéndose 
Perelman, C. (2021). Nuevo código de puntuación de Gimnasia Artística Femenina. Federación Internacional de Gimnasia. Ciclo Olímpico $2022-2024$. Revista Ciencias de la Actividad Física UCM, N²2(1), enero-junio, 1-10. DOI: http://doi.org/10.29035/rcaf.22.1.4

específicamente a Campeonatos Mundiales y JJOO. En el COP actual, los Paneles de Jueces están conformados por 9 jueces, pero divididos en 2 jueces $D$, 5 jueces E y 2 jueces R (Referencia). Es decir, para el próximo ciclo se eliminan los jueces de Referencia.

\section{Funciones del Jurado del Aparato:}

Actualmente, el Panel D controla que se cumpla el tiempo o cantidad de ensayos de calentamiento de las gimnastas en el aparato Salto. En el nuevo COP esta función la cumplirá el Juez de Línea del aparato, bajo supervisión del Panel D. El juez de línea deberá contar la cantidad de intentos de calentamiento permitidos según la etapa de competencia.

\section{Tiempos}

El tiempo relacionado con todos los ejercicios (incluido el inicio del ejercicio, la duración del ejercicio, etc.), tendrá un incremento de 1 segundo en el nuevo COP.

\section{Parte II. Evaluación de los ejercicios}

\section{Sección 6: Determinación de la Nota}

\section{Nota Final (NF):}

El cálculo de la Nota Final de la gimnasta se hace sumando dos notas separadas, la Nota D y la Nota E. (FIG, 2017). Los dos jueces D determinan en conjunto una sola Nota D, nota que da cuenta del contenido del ejercicio. Los cinco jueces E calculan sus descuentos y ponen su nota en forma independiente. Para el cálculo de la Nota E, se borra la nota más alta y la más baja de los jueces $\mathrm{E}$ y se promedian las tres notas intermedias. Luego este promedio se resta de 10,00 ptos. y se obtiene la Nota E.

Para el nuevo COP se proponen siete jueces E, versus los cinco jueces de E que son actualmente. La propuesta para determinar la Nota E para el siguiente ciclo olímpico, es que se promedien las tres notas intermedias; borrando los dos descuentos más altos y los dos descuentos más bajos.

\section{Sección 7: Reglas para la Nota D Valor de Dificultad (VD):}

"EI VD son los elementos de la Tabla de Elementos del COP, la cual es abierta y puede ampliarse_según sea necesario" (FIG, 2017, p.1). Actualmente los VD de los elementos van de la letra A a la letra I, siendo el valor de $A=0,10$ ptos. (el más bajo) y el valor de I = 0,90 ptos. (el más alto).

Para el nuevo COP se incluye dentro de los VD, los elementos de valor J, cuyo valor es de 1,00 pto. Esto obedece a que, cada cierto tiempo se incorporan elementos nuevos ejecutados en eventos internacionales oficiales al COP. El Comité Técnico Femenino de la FIG otorga el valor correspondiente al nivel de dificultad del elemento nuevo presentado, encontrándose a partir de ahora con elementos que se les han reconocido con la letra J. Por consecuencia, los VD en el nuevo COP irán de la letra A a la letra J.

\section{Reconocimiento del VD de los elementos:}

Los elementos se reconocen como iguales o diferentes, de acuerdo a diferentes parámetros establecidos en el COP. Uno de estos parámetros es que los elementos reciben VD sólo una vez y en orden cronológico. Respecto a este punto, el nuevo COP limita la cantidad de giros de danza de la siguiente manera: "Solo se reconocerá un giro o pirueta de cada línea (familia) de la Tabla de Elementos". Con esto se quiere evitar que la gimnasta presente sólo un tipo de giro varias veces durante su rutina para el VD, cambiando sólo el grado de giro y no la familia del giro. Esta nueva regla busca que haya variedad en la selección de elementos de la rutina.

En relación a los elementos acrobáticos, éstos se consideran diferentes si aparecen con el mismo número en la Tabla de Elementos y siguen los siguientes criterios:

- Diferente posición del cuerpo en mortales (agrupada, carpada o extendida).

- Diferente grado de giro: $1 / 2,1 / 1,11 / 2\left(180^{\circ}, 360^{\circ}\right.$, $\left.540^{\circ}\right)$, etc.

- El apoyo se realiza sobre uno o ambos brazos o libre (sin manos). 
Perelman, C. (2021). Nuevo código de puntuación de Gimnasia Artística Femenina. Federación Internacional de Gimnasia. Ciclo Olímpico $2022-2024$. Revista Ciencias de la Actividad Física UCM, N²2(1), enero-junio, 1-10. DOI: http://doi.org/10.29035/rcaf.22.1.4

- El despegue se realiza con una o ambas piernas.

Nuevo: Para Paralelas Asimétricas, los mortales con piernas juntas y separadas se considerarán elementos diferentes.

\section{Valor de Enlace (VE):}

El VE se logra mediante combinaciones únicas de elementos en Paralelas Asimétricas, Viga y Suelo (FIG, 2017). Actualmente los VE pueden ser de 0,10 o 0,20 ptos., y es posible llegar a 0,30 ptos. en Viga por el Bono por Serie (BSE) que vale 0,10 pts. Cabe señalar que los VE sólo se otorgan si no hay caída.

Para el nuevo COP se agrega dentro de los VE, un Bono por Salida (BSA) de 0,20 ptos. en Paralelas Asimétricas, Viga y Suelo; que se otorgará para las salidas de Valor D o más. Para recibir este Bono, siguiendo la lógica existente, la salida deberá ejecutarse sin caída.

\section{Sección 8: Reglas para la Nota E (Ejecución)}

“Con perfección en la ejecución, combinación y presentación artística, la gimnasta puede obtener una nota máxima de 10.00 ptos." (FIG, 2017, p. 1) en Ejecución. La Nota E incluye las deducciones por faltas de Ejecución y Presentación Artística. "Las deducciones por errores de Ejecución y Artístico se suman, y luego se restan de 10.00 pts. para determinar la Nota E" (FIG, 2017, p. 1) de cada juez.

\section{Tabla de Faltas y Penalizaciones Generales:}

La Tabla de Faltas y Penalizaciones Generales del cop da cuenta de todos los tipos de faltas que existen con sus respectivos descuentos, donde cada falta se divide en falta leve (0,10 ptos.), mediana $(0,30$ ptos.), grave (0,50 ptos.) o muy grave (1,00 pto. o más).

Esta tabla también indica qué tipo de jueces o paneles de jueces son los responsables de aplicar cada uno de los descuentos.

Para ello, las faltas en esta tabla se encuentran divididas en:

- Faltas de Ejecución (Panel E).
- Faltas de Dificultad (Panel D).

- Irregularidades en los Aparatos (Panel D y Jurado Superior).

- Faltas de comportamiento de la Gimnasta (Jurado Superior).

- Faltas de comportamiento del Entrenador (Jurado Superior).

En la Tabla de Faltasy Penalizaciones Generales del nuevo COP se encuentran faltas nuevas y cambios en los descuentos:

Nuevo: Insuficiente exactitud de la posición carpada en dobles mortales.

Ángulo de cadera > a 90, falta leve = descuento 0,10 ptos. (Panel E).

Nuevo: En la recepción, rozar/tocar el aparato/colchoneta con las manos, pero sin caída. Falta media $=0,30$ ptos. cada vez (Panel E).

Nuevo: Realizar la salida con caída. No se otorga Bono por Salida. (Panel D).

Cambio: Paso o salto muy grande en la recepción, más del ancho de hombros.

En el Código de Puntuación actual, la guía es más de 1 metro (Panel E).

\section{Sección 9: Directivas Técnicas}

Posiciones del cuerpo y Reconocimiento del elemento:

En mortales extendidos, “... la mayor parte del mortal se debe realizar en posición extendida" (FIG, 2017, p.1) para que sea reconocido como tal. Esto es válido para mortales simples o dobles mortales. "Si no se muestra la posición extendida, se considera posición carpada" (FIG, 2017, p.1) en los elementos sin giro en el eje longitudinal, en Paralelas Asimétricas, Viga y suelo.

En el nuevo COP se permitirá en Salto una leve flexión de cadera (hasta $30^{\circ}$ ) en los mortales extendidos sin giro en el eje longitudinal, para ser reconocido como extendido.

Actualmente, una leve flexión de cadera en Salto hace que el mortal sea considerado como carpado, lo que hace muy difícil determinar el límite en 
Perelman, C. (2021). Nuevo código de puntuación de Gimnasia Artística Femenina. Federación Internacional de Gimnasia. Ciclo Olímpico $2022-2024$. Revista Ciencias de la Actividad Física UCM, N²2(1), enero-junio, 1-10. DOI: http://doi.org/10.29035/rcaf.22.1.4

que se debe considerar un mortal como extendido o carpado (Panel D).

Valores de Dificultad (VD) de saltos de danza con giro:

En el COP actual se permiten diversas técnicas para los saltos de danza con un giro de $360^{\circ}$ o más en el eje longitudinal en Suelo y Viga. Las posiciones carpadas, agrupadas “... y con piernas separadas de los saltos se pueden hacer al comienzo, en el medio o al final del giro" (FIG, 2017, p.2).

En el nuevo COP se agrega al punto anterior, que para los saltos de danza con medio giro (180), la posición prescrita tiene que ser ejecutada al comienzo o al final del salto para recibir el VD, y no al medio.

Dentro de esta misma sección, actualmente si faltan más de $30^{\circ}$ de giro en el eje longitudinal en un salto de danza, "... se reconoce como otro elemento del Código. La posición de los hombros y de la cadera es decisiva" (FIG, 2017, p.2).

En el nuevo COP se complementa el punto anterior, indicando que, para los saltos de danza con aterrizaje en dos pies, el pie delantero es decisivo.

\section{Requisitos para elementos de danza específicos:}

Para los saltos Zancada a anillo, Zancada con cambio a anillo y Zancada desde dos piernas a anillo (con/sin giro), los requerimientos técnicos de acuerdo al FIC (2017) son:

Arco del tronco y cabeza atrás.

Split $180^{\circ}$ de piernas.

Pierna de adelante a la horizontal y pie de atrás a la coronilla.

El giro se debe realizar después de mostrar la posición anillo (p. 4).

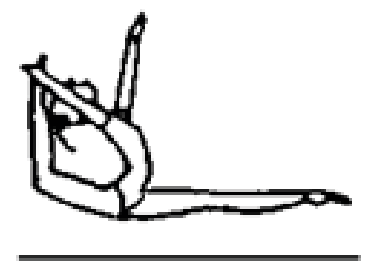

Figura 7. Fuente: FIG (2017).
Para el nuevo COP, se agrega el requisito de pierna de atrás flexionada $\left(135^{\circ}\right)$. Y en relación con este requisito, si la gimnasta realiza el salto con la pierna de atrás extendida, el Panel E hará un descuento de 0,10 ptos. por falta leve.

\section{Parte III. Aparatos}

\section{Sección 10: Salto}

El COP clasifica los saltos en 5 grupos, los cuales se mantienen para el próximo ciclo olímpico:

Grupo 1: Saltos sin mortal (Inversión adelante 0 Handvolt, Yamashita, Rondat) con o sin giro en el eje longitudinal (EL) en el 1er y/o 2do vuelo.

Grupo 2: Inversión adelante o Handvolt con o sin giro $\left(360^{\circ}\right)$ en el EL en el Ter vuelo - mortal adelante o atrás con o sin giro en el EL en el 2do vuelo.

Grupo 3: $\quad$ Inversión adelante con $1 / 4-1 / 2$ giro $\left(90^{\circ}\right.$ - $\left.180^{\circ}\right)$ en el EL en el ler vuelo (Tsukahara) - mortal atrás con o sin giro en el EL en el 2do vuelo.

Grupo 4: $\quad$ Rondat (Yurchenko) con o $\sin 3 / 4$ de giro $\left(270^{\circ}\right)$ en el EL en el ler vuelo - mortal atrás con o sin giro en el EL en el 2do vuelo.

Grupo 5: Rondat con $1 / 2$ giro $\left(180^{\circ}\right)$ en el EL en el Ter vuelo - mortal adelante o atrás con o sin giro en el EL en el 2do vuelo (FIG, 2017, p. 1).

\section{Deducciones Específicas del Aparato - Panel E (Ejecución)}

Los descuentos específicos de este aparato se dividen de acuerdo a las fases 4 del salto, que son las siguientes: primera fase de vuelo, fase de repulsión, segunda fase de vuelo y aterrizaje.

En relación a la "Falta de Grados de Giro en el EL en la Primera Fase de Vuelo", el COP actual contempla lo siguiente: 
Perelman, C. (2021). Nuevo código de puntuación de Gimnasia Artística Femenina. Federación Internacional de Gimnasia. Ciclo Olímpico $2022-2024$. Revista Ciencias de la Actividad Física UCM, N²2(1), enero-junio, 1-10. DOI: http://doi.org/10.29035/rcaf.22.1.4
- Para el Grupo 1 con $1 / 2$ giro $\left(180^{\circ}\right)$
$\leq 45^{\circ}$, descuento 0,10 ptos. (falta leve).
$\leq 90^{\circ}$, descuento 0,30 ptos. (falta media).
- Para el Grupo 4 con 3/4 giro $\left(270^{\circ}\right)$
$\leq 45^{\circ}$, descuento 0,10 ptos. (falta leve).
- Para el Grupo 1 o 2 con 1 giro $\left(360^{\circ}\right)$
$\leq 45^{\circ}$, descuento 0,10 ptos. (falta leve).
$\leq 90^{\circ}$, descuento 0,30 ptos. (falta media).
$>90^{\circ}$, descuento 0,50 ptos. (falta grave)

En el nuevo COP se agregó la siguiente falta:

Para el Grupo 3 de Salto con $1 / 4$ giro $\left(90^{\circ}\right)$

$\leq 45^{\circ}$, descuento 0,30 ptos. (falta media).

En relación a la "Falta de Posición del Cuerpo durante la Segunda Fase de Vuelo", específicamente referido a las posiciones extendidas en mortales, el COP actual no permite ángulo de cadera en los mortales extendidos. Un leve ángulo de cadera en el mortal extendido trae como consecuencia que el salto sea considerado como carpado por el Panel D, con los correspondientes descuentos por parte del Panel E por no mantener la alineación del cuerpo extendido o por no mostrar exactitud en la posición carpada, dado que el salto fue considerado carpado y no extendido.

Dentro de los cambios propuestos en la Sección de Directivas Técnicas del nuevo COP, se advierte que se permitirá en Salto una leve flexión de cadera (hasta $30^{\circ}$ ) en los mortales extendidos sin giro en el EL, para ser reconocidos como extendidos. Es por esto que se agregó en el nuevo COP, en la Tabla de Faltas Específicas de Salto, la falta de alineación del cuerpo en mortal extendido sin giro en el eje longitudinal en el segundo vuelo, con los siguientes descuentos:

- Leve flexión de cadera, descuento 0,10 ptos. (falta leve).

- Más de $30^{\circ}$ de flexión de cadera, descuento 0,30 ptos. (falta media).

\section{Sección 17: Paralelas Asimétricas Valor de Enlace (VE):}

EI VE se puede otorgar por enlaces directos, donde ambos elementos deben tener vuelo o mínimo 1/2 giro $\left(180^{\circ}\right)$ en el EL. El VE forma parte de la Nota D, por lo que es responsabilidad del Panel D (tablas 2 y 3).

Tabla 2

En el COP actual, los VD se dan de acuerdo a las siguientes fórmulas.

\begin{tabular}{|c|c|}
\hline 0,10 pts & 0,20 ptos. \\
\hline \multirow{2}{*}{$\begin{array}{l}D+D \\
\text { (o más) }\end{array}$} & $\begin{array}{c}\text { D (vuelo misma banda o de barra inferior a barra superior) + C o más (en barra superior) - se } \\
\text { deben realizar en ese orden }\end{array}$ \\
\hline & D + E (ambos elementos con vuelo) \\
\hline
\end{tabular}

Fuente: FIG, (2017).

Tabla 3

En el nuevo COP, la propuesta para las fórmulas para VE es la siguiente:

\begin{tabular}{|c|c|}
\hline 0,10 pts & 0,20 pts \\
\hline \multirow[t]{3}{*}{$\begin{array}{c}\text { D + D } \\
\text { (o más) }\end{array}$} & $\begin{array}{c}\text { D (vuelo misma banda o de barra inferior a barra superior) + C o más (en barra superior) } \\
\text { - se deben realizar en ese orden }\end{array}$ \\
\hline & $\mathrm{E}+\mathrm{E}$ (un elemento debe tener vuelo) \\
\hline & $F+D(a m b o s$ elementos deben tener vuelo) \\
\hline
\end{tabular}

Fuente: FIG, (2021) 
Perelman, C. (2021). Nuevo código de puntuación de Gimnasia Artística Femenina. Federación Internacional de Gimnasia. Ciclo Olímpico $2022-2024$. Revista Ciencias de la Actividad Física UCM, N²2(1), enero-junio, 1-10. DOI: http://doi.org/10.29035/rcaf.22.1.4

Para reconocer los VE en Paralelas Asimétricas, los elementos se deben enlazar en forma directa. No se permiten Impulsos Suplementarios, que "... son impulso de piernas desde apoyo facial y/o balanceos no necesarios, que se hacen para poder realizar el siguiente elemento" (FIG, 2017, p. 2). Tampoco se permiten Impulsos Vacíos, que se refieren “... a balanceo adelante o atrás sin ejecución de un elemento de la Tabla antes de que el balanceo vuelva en sentido contrario" (FIG, 2017, p. 2).

En el COP actual se especifica que, "si entre dos elementos se realiza un Impulso Vacío o un Impulso Suplementario, no se puede otorgar el VE" (FIG, 2017, p. 2).

En el nuevo COP se complementa la aclaración anterior, agregando que no se puede otorgar VE si además después del segundo/último elemento se realiza un Impulso Vacío o un Impulso Suplementario.

\section{Deducciones Específicas del Aparato - Panel E:}

En relación a la Tabla Deducciones Específicas de las Paralelas Asimétricas, el nuevo COP incorpora la siguiente falta de ejecución con su descuento correspondiente:

- Rozar la colchoneta 0,30 ptos. (falta media).

\section{Sección 12: Viga de Equilibrio}

\section{Requisitos de Composición (RC):}

Existen cuatro RC en cada aparato, excepto en Salto. Cada uno vale 0,50 pts., lo que da un total 2,00 pts., que forman parte de la Nota D.

Los RC en Viga actualmente son:

1. Un enlace de por lo menos 2 elementos de danza diferentes, donde uno de los saltos debe mostrar separación de piernas de $180^{\circ}$

2. Un Giro del Grupo 3.

3. Una serie acrobática de mínimo 2 elementos con vuelo (con o sin apoyo de manos), donde uno es 1 mortal.
4. Elementos acrobáticos en diferentes direcciones (ad/lat y atrás).

Para el nuevo COP, el segundo RC de "Giro del Grupo 3", se ha modificado. La gimnasta podrá hacer un giro de danza o un elemento del grupo de las volteretas (Grupo 4), para cumplir con este requisito.

\section{Valor de Enlace (VE) y Bonificación por Serie (BS):}

En relación a este punto, el código actual (FIG, 2017) plantea lo siguiente:

- EI VE se puede otorgar por enlaces directos.

- VE se suma en la Nota D.

- La BS se obtiene por el enlace de 3 mínimo elementos.

Para el nuevo COP se suma a lo anterior, que:

- No se dará VE, ni Bono por Serie (BS), si la gimnasta agarra la Viga para evitar una caída.

\section{Fórmulas para enlaces directos:}

Para otorgar Valor de Enlace (VE) de elementos acrobáticos enlazados directamente, la exigencia actual es que sean 2 elementos acrobáticos con vuelo y con rebote; incluidas las entradas y salidas mínimo F. En el nuevo COP se excluyen las salidas para esta fórmula de enlaces.Sección 13: Suelo

En el nuevo COP, todos los tiempos se han incrementado en 1 segundo. Por lo que el descuento por tiempo largo de la rutina se aplicará, si la duración de la rutina es mayor a 1:31 minutos (91 segundos), con un descuento de 0,10 ptos. de la Nota Final. 
Perelman, C. (2021). Nuevo código de puntuación de Gimnasia Artística Femenina. Federación Internacional de Gimnasia. Ciclo Olímpico $2022-2024$. Revista Ciencias de la Actividad Física UCM, N²2(1), enero-junio, 1-10. DOI: http://doi.org/10.29035/rcaf.22.1.4

\section{CONCLUSIONES}

La Gimnasia Artística es un deporte de apreciación evaluado por jueces. En este sentido, la FIG persigue constantemente la objetividad y consenso en las evaluaciones de los jueces; y que gane la mejor gimnasta independiente del país al que represente. Asimismo, la FIG actualiza el COP para cada ciclo olímpico, con el fin de adecuarse a los cambios que va sufriendo la implementación de este deporte y al nivel de las nuevas dificultades que presentan las gimnastas, que es cada día más alto. Con las modificaciones del COP se pretende también ir variando los elementos que escogen las gimnastas para construir sus rutinas, bajando el valor de elementos o enlaces que son ejecutados por muchas gimnastas y/o subiendo el de elementos y enlaces poco seleccionados, buscando con esto la diversidad y originalidad en la composición de las rutinas.

Por otro lado, muchos cambios en el COP apuntan a evitar riesgos y lesiones en los gimnastas y, de acuerdo a las estadísticas, se van eliminado elementos o enlaces que pueden poner en peligro la salud de los deportistas.

Como el reglamento de este deporte es variable, es necesario que los jueces, técnicos y gimnastas conozcan y sepan aplicar en forma correcta el COP. En este sentido, mientras antes se pueda tener la información de los cambios del COP que emanan de la FIG, se pueden planificar los entrenamientos y adaptar las rutinas con más tiempo, convirtiéndose esto en una ventaja competitiva. El objetivo de este trabajo fue dar a conocer con tiempo los cambios que se están proponiendo para el nuevo COP, y así no tener que esperar hasta después de los JJOO de Tokio 2022 para que salga el Código nuevo definitivo; por el contrario, el propósito es entregar la información con antelación para todos los relacionados con la disciplina.

\section{REFERENCIAS BIBLIOGRÁFICAS}

Fédéraction International de gymnastique (2017). 2017 - 2020 Code of Points. Women's Artistic Gymnastics. https://eugymnastics.files.wordpress.com/2017/04/cop_wag_20172020_ici-el.pdf

Fédéraction International de gymnastique. (2018). Newsletters. The women's artistic gymnastics newsletter - $n^{\circ}$ 43. https://www.9ymnastics.sport/site/pages/newsletterswag.php

Fédéraction International de gymnastique. (2019). Newsletters. The women's artistic gymnastics newsletter - $n^{\circ}$ 44. https://www.gymnastics.sport/site/pages/newsletterswag.php

Fédéraction International de gymnastique. (2021). 2021-2024 Code of points, Women's Artistic Gymnastics. https://gimnasialatina.com/wp-content/uploads/2019/11/WAG_CoP_20212024_draft_Parts_Sept_2019_pdf.pdf

Gimnasialatina. (2019). Borrador Código GAF 2021 2024. GimnasiaLatina. https://gimnasialatina.com/borrador-codigo-gaf-2021-2024/

Perelman, C. (2020). Gymnastics Interactive Program [Software]. https://www.websts.com/index.php

Sacchi, D., Dowdell, K., Zhou, Q., Andrianova, L., Davydova, E., Gratt, J., Perskaia, T., Chusovitina, O., \& Valenzo, N. (2019). WAG Help Desk Edition $\quad 7 . \quad$ https://www.gymnastics.sport/publicdir/rules/files/en_WAG\%2OHelp\%2ODesk\%2O(Edition\%207)\%20.pdf 
Perelman, C. (2021). Nuevo código de puntuación de Gimnasia Artística Femenina. Federación Internacional de Gimnasia. Ciclo Olímpico $2022-2024$. Revista Ciencias de la Actividad Física UCM, N²2(1), enero-junio, 1-10. DOI: http://doi.org/10.29035/rcaf.22.1.4

\section{Dirección para correspondencia}

Mg. Claudia Marcela Perelman B arry

Universidad Metropolitana de Ciencias de la Educación

Santiago, Chile.

Dirección postal: Av. José Pedro Alessandri 774

ORCID: https://orcid.org/0000-0003-3997-6855

Contacto:

claudia.perelman@umce.cl

Recibido: 24-09-2020

Aceptado: 28-01-2021

@๑ఠ

Este obra está bajo una Licencia de Creative Commons ReconocimientoNoComercial-Compartirlgual 4.0 Internacional. 